\title{
Herausforderungen bei der Entwicklung neuer Psychopharmaka
}

Psychische Leiden sollten eigentlich ein attraktives Indikationsgebiet für forschende Pharmafirmen sein: Es gibt einen hohen, teilweise ungedeckten medizinischen Bedarf. Weltweit steigen die Fallzahlen und die stationären Behandlungsfälle. Die Weltgesundheitsorganisation WHO vermeldet, dass mehr als 300 Mio. Menschen aller Altersklassen an Depressionen leiden, die wiederum die Hauptursache für Einschränkungen bzw. Arbeitsunfähigkeit sind und erheblich zur globalen Krankheitslast beitragen. 800.000 Personen begehen jährlich Suizid, wobei dies die zweithäufigste Todesursache bei den 15-29 Jährigen ist. Trotz verfügbarer effektiver Behandlungen werden im Durchschnitt weniger als die Hälfte der Betroffenen therapiert, in manchen Ländern sogar weniger als 10\% [1].

Dennoch sind die Forschungs- und Entwicklungsaktivitäten der Pharmafirmen in diesem Gebiet vergleichsweise gering [2]. Dies hat insbesondere folgende Gründe:

- keine guten Krankheitsmodelle verfügbar: nur wenige validierte Biomarker und Proof-of-Concept-Modelle

- breite Verwendung von subjektiven Diagnostik-Skalen und subjektiven primären Endpunkten: daraus resultiert hohe Placebo-Response

- geringe Erfolgsquote: lediglich 8\% der Wirkstoff-Kandidaten, die es in die klinische Prüfung schaffen, erreichen die Zulassung; viele Projekte scheitern in der späten Entwicklung. Die Erfolgsquote in klinischen Prüfungen der Phase II betrug 2006-2015 lediglich 24\% zusammen mit Herz-Kreislauf-Medikamenten war dies der letzte Platz über alle Indikationsbereiche.

- Medizinprodukte und IT-Lösungen: „Electroceuticals“ wie Hirnschrittmacher, Elektrostimulation, Computer-gestützte Anti-Depressions-Programme.

Hinzu kommen weitere Hürden wie längere Entwicklungs- und Zulassungszeiten, Probleme in den Nutzenbewertungen der verschiedenen Länder wegen der subjektiven Wirksamkeitskriterien, die Einstufung von psychischen Erkrankungen als „Lifestyle"-ähnliche Beeinträchtigungen und das hohe Wechselwirkungspotenzial, das zusammen mit Nebenwirkungen (starke Gewichtszunahme, Libidoverlust, Impotenz) zu einer schlechten Adhärenz beiträgt.

All dies hat dazu geführt, dass sich einige große Firmen in den letzten Jahren aus dem Gebiet der psychischen Krankheiten zurückgezogen haben und von den fortgeschrittenen Entwicklungsprojekten für neue Therapien weniger als $2 \%$ solche $\mathrm{Er}$ krankungen betreffen; auf Krebserkrankungen entfallen hingegen mehr $40 \%$ und auf Entzündungskrankheiten mehr als 15\% der Projekte [3].

Seit 2011 sind nur wenige Präparate mit neuem Wirkstoff gegen Depressionen und Schizophrenie zugelassen und in Deutschland vermarktet worden. Und von diesen wurden 2 nach nicht zufriedenstellenden Nutzenbewertungen wieder vom Markt genommen [4]. Gegen Depressionen sind lediglich 7 Projekte in Phase III und 8 in Phase II der klinischen Entwicklung, gegen Schizophrenie sogar nur 3 in Phase III und 8 in Phase II. Bei Angststörungen sind es 1 in Phase III und 3 in Phase II [5]. Dem standen z.B. 2016 im Bereich Onkologie 237 Projekte in Phase III und 721 Projekte in Phase II gegenüber [6].

Doch Hoffnung auf neuen Schub für die Psychopharmaka-Entwicklung machen neue Erkenntnisse:

- Mit Genomik-Technologien konnten zunehmend Allele identifiziert werden, die mit Schizophrenie und bipolaren Störungen assoziiert sind.

- Durch die Aufklärung molekularer Wege können neue Angriffspunkte ermittelt werden.

- Mit Bild-gebenden Verfahren wie MRT und PET lassen sich Diagnosen verbessern und das Ansprechen auf neue Therapieansätze verfolgen.

- Letztendlich soll die Therapie psychischer Krankheiten - wie bei Krebserkrankungen zunehmend der Fall personalisiert werden [7].

$\mathrm{Zu}$ diesen Fortschritten haben insbesondere auch die Aktivitäten beim IMI (Innovative Medicines Initiative) Projekt
NEWMEDS beigetragen. Dieses von 2009-2015 durchgeführte Projekt hat folgende Ergebnisse gebracht:

- pharmakologische Validierung einer Spezies-übergreifenden „Imaging Battery“ für die Arzneimittelentwicklung

- genetische Varianten, die mit Schizophrenie und Autismus assoziiert sind, haben Impact auf kognitive Fähigkeiten und Hirnstrukturen bei nicht erkrankten Trägern

- neue Einsichten in die Biologie der Schizophrenie und Depression, insbesondere die thalamo-kortikalen und präfrontalen Cortex-hippocampalen Schaltkreise bei Tiermodellen

- Methodologie zur PET-Messung von Änderungen der GABA-Spiegel mittels neuer Radioliganden (für Entwicklung/ Monitoring neuer Psychopharmaka)

- frühe Schizophrenie-Studien können kürzer u. effizienter werden durch Einbeziehung von mehr Frauen und Personen mit bestimmten Symptomen und/oder jüngeren Patienten

- phänotypische Charakterisierung und pharmakologische Validierung von 3 Mausstämmen mit den gleichen Autismus-/Schizophrenie-assoziierten Genvarianten wie bei Trägerpersonen

- Anwendung von Maschinenlernmethoden auf kritische Fragen bei der Arzneimittelentwicklung (Stratifizierung des Ansprechens; Überwindung der BlutHirn-Schranke und Lokalisation der Effekte; Abschätzung von Stärke und Ort der Wirkstoff-Effekte)

- Standardisierung von Touchscreen-Methodologien und kognitiven Modellen über mehrere Unternehmen hinweg [8].

Hinzu kommen Forschungsprogramme auf nationaler Ebene (Forschungsnetz psychische Erkrankungen; Laufzeit 20152019) [9], das starke Engagement einiger Firmen und künftig vielleicht Erkenntnisse aus dem EU Human Brain Project (20132023) [10], das mit rund 1,2 Mrd. Euro gefördert wird und u.a. Erkenntnisse über Hirnerkrankungen generieren soll.

Die ähnlich ausgerichtete U.S. BRAIN Initiative ist 2013 u.a. von dem Neuro-Wis- 
senschaftler und Psychiater Prof. Dr. Karl Deisseroth/Standford University initiiert worden; Deisseroth erhielt 2017 den mit 4 Mio. Euro dotierten Else Kröner Fresenius Preis für seine Forschungsarbeiten im Bereich Optogenetik und Sichtbarmachung von Nervenzellnetzwerken, gerade auch bei Depressionen. Er arbeitet daran, die Ursachen für Depressionen aufzuklären und Therapien zu entwickeln. Im Vorfeld der Preisverleihung war 2014 beim Nobelpreisträgertreffen in Lindau das Thema „The biological basis of psychiatric disorders" als dasjenige ermittelt worden, das die größten Fortschritte in den nächsten Jahren verspricht [11].

\section{Fazit}

Bei der Entwicklung neuer Psychopharmaka gegen Schizophrenie und Depressionen sind besonders viele Herausforderungen zu meistern - bei den präklinischen Modellen, der Diagnose, der Heterogenität der Patienten, der Dauer der Studien, den Endpunkten, den Anforderungen an die Zulassung und danach an die Nutzenbewertungen sowie bei der allgemeinen Einschätzung von Psychopharmaka. An diesen Hürden sind viele Projekte gescheitert, und einige Firmen haben ihr Engagement in diesem Bereich reduziert oder eingestellt.
Dessen ungeachtet gibt es aber nach wie vor eine Reihe von Projekten in Phase II und III oder im Zulassungsverfahren.

Neue Forschungsergebnisse - auch aus Public Private Partnerships wie der Innovative Medicines Initiative - lassen einen Schub bei der Entwicklung von Medikamenten gegen diese Krankheiten erwarten.

\section{Interessenkonflikte}

Der Autor ist Geschäftsführer Forschung, Entwicklung, Innovation im vfa, dem Wirtschaftsverband der forschenden PharmaUnternehmen in Deutschland.

Autor

Siegfried Throm

Geschäftsführer Forschung, Entwicklung, Innovation im vfa, Berlin

\section{Korrespondenzadresse}

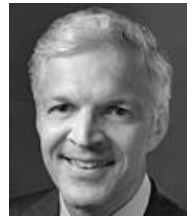

Dr. Siegfried Throm

Geschäftsführer Forschung,

Entwicklung, Innovation

vfa

Hausvogteiplatz 13

10117 Berlin

s.throm@vfa.de

\section{Literatur}

[1] WHO. Factsheet Depression (Februar 2017). Im Internet: http://www.who.int/mediacentre/factsheets/fs369/en/; Stand: 15.08.2017
[2] MacEwan JP, Seabury S, Aigbogun MS et al. Pharmaceutical Innovation in the Treatment of Schizophrenia and Mental Disorders Compared with Other Diseases (Aug 2016). Im Internet: https://www.ncbi.nlm.nih.gov/pmc/ articles/PMC5022985/; Stand: 15.08.2017

[3] Forschung für das Leben. Berlin: vfa; 2017

[4] Gründer G. Bedeutung des Arzneimittelmarktneuordnungsgesetzes für die Psychopharmakotherapie. Nervenarzt 2016; 87: 356-366

[5] PhRMA compilation of 2016 Medicines in Development for Mental Health (22.04.2016). Im Internet: http://phrmadocs.phrma.org/sites/default/files/pdf/ medicines-in-development-drug-listmental-illnesses.pdf; Stand: 15.08.2017

[6] Scrip intelligence. Informa UK; 3808: 5; 24. Juni 2016

[7] Caracia F, Leggio GM, Salomone S. et al. New drugs in psychiatry: focus on new pharmacological targets (March 2017). Im Internet: https://www.ncbi.nlm.nih.gov/pmc/articles/ PMC5373420/; Stand: 15.08.2017

[8] IMI Annual Activity Report 2015 (28.06.2016). Im Internet: https://www.imi. europa.eu/sites/default/files/uploads/documents/Governance/IMI_AAR2015.pdf; Stand: 15.08 .2017

[9] Forschungsnetz psychische Erkrankungen (03.07.2017). Im Internet: https://www. gesundheitsforschung-bmbf.de/de/ Forschungsnetz-psychische-Erkrankungen. php; Stand: 15.08.2017

[10] https://www.humanbrainproject.eu/en/; Stand: 15.08 .2017

[11] https://www.ekfs.de/en/scientific-funding/ research-prize-2017/; Stand: 15.08.2017 